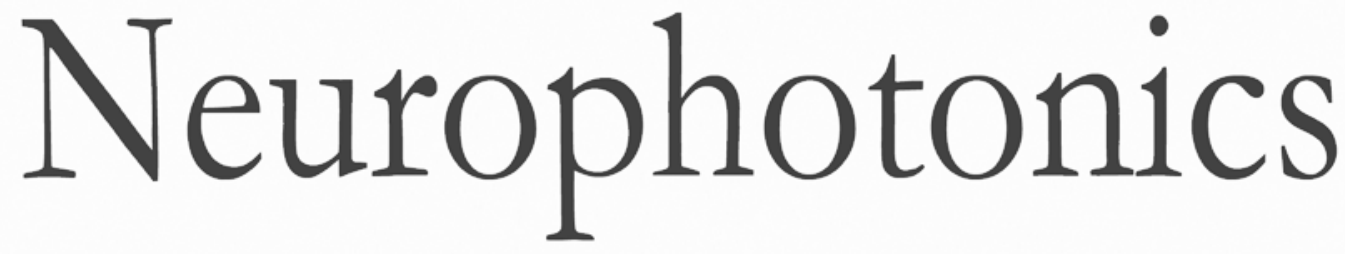

\title{
Cerebral edema detection in vivo after middle cerebral artery occlusion using swept-source optical coherence tomography
}

\author{
Jian Liu \\ Yan Li \\ Yao $\mathrm{Yu}$ \\ Xincheng Yuan \\ Hongyu Lv \\ Yuqian Zhao \\ Zhenhe Ma
}




\title{
Cerebral edema detection in vivo after middle cerebral artery occlusion using swept-source optical coherence tomography
}

\author{
Jian Liu, ${ }^{a}$ Yan Li, ${ }^{a}$ Yao Yu, ${ }^{a}$ Xincheng Yuan, ${ }^{b}$ Hongyu Lv, ${ }^{c}$ Yuqian Zhao, ${ }^{a}$ and Zhenhe Ma ${ }^{a, *}$ \\ ${ }^{a}$ Northeastern University at Qinhuangdao, School of Control Engineering, Qinhuangdao, China \\ bUniversity of Michigan, Department of Biomedical Engineering, Ann Arbor, Michigan, United States \\ cMaternal and Child Health Hospital, Department of Ophthalmology, Qinhuangdao, China
}

\begin{abstract}
Cerebral edema is a severe complication of ischemic cerebrovascular disease, which can lead to microcirculation compression resulting in additional ischemic damage. Real-time and continuous in vivo imaging techniques for edema detection are of great significance to basic research on cerebral edema. We attempted to monitor the cerebral edema status in rats with middle cerebral artery occlusion (MCAO) over time, using a wide field-of-view swept-source optical coherence tomography (SS-OCT) system. Optical attenuation coefficients (OACs) were calculated by an optimized depth-resolved estimation method, and en face OAC maps covering the whole cortex were obtained. Then, the tissue affected by edema was segmented from the OAC maps, and the cortical area affected by edema was estimated. Both magnetic resonance image (MRI) and brain water content measurements were used to verify the presence of cerebral edema. The results showed that the average $\mathrm{OAC}$ of the ischemic area gradually decreased as cerebral edema progressed, and the edema area detected by SS-OCT had high similarity in position and shape to that obtained by MRI. This work extends the application of OCT and provides an option for detecting cerebral edema in vivo after ischemic stroke. ๑ The Authors. Published by SPIE under a Creative Commons Attribution 4.0 Unported License. Distribution or reproduction of this work in whole or in part requires full attribution of the original publication, including its DOI. [DOI: 10.1117/1.NPh.6.4.045007]
\end{abstract}

Keywords: swept-source optical coherence tomography; cerebral edema; middle cerebral artery occlusion; optical attenuation coefficient; wide field-of-view.

Paper 19059RR received Jun. 12, 2019; accepted for publication Oct. 14, 2019; published online Nov. 7, 2019.

\section{Introduction}

Ischemic cerebrovascular disease (ICVD) is characterized by high morbidity, high disability, high recurrence rate, and high mortality. ${ }^{1}$ Cerebral edema is a severe complication of ICVD. ${ }^{2}$ Recent studies have shown that cerebral edema can independently impair cerebral metabolism and may cause compression of microcirculation, which can lead to additional ischemic damage. ${ }^{3}$

At present, clinical treatments of cerebral edema mainly include dehydrating agent treatment and surgical treatment. ${ }^{4}$ Decompressive craniectomy reduces the risk of high intracranial pressure (ICP) by removing a bone flap covering swollen brain tissue in order to provide space for the expansion of the swollen brain. Although it significantly reduces the number of deaths or severe disabilities in patients with malignant middle cerebral artery (MCA) stroke, ${ }^{5}$ this treatment is also a highly invasive procedure with certain risks. ${ }^{6}$ Dehydrating agents such as mannitol reduce the amount of cerebral water after osmotic dehydration. However, if the method adopted or treatment timing is not appropriate, the progression of cerebral edema will not be alleviated or may even be worsened. ${ }^{7}$ Therefore, a safer and more effective alternative is necessary for the treatment of this disease. Basic research on cerebral edema using animal models is very useful for understanding the formation mechanism of cerebral edema and evaluating the effectiveness of treatment strategies.

*Address all correspondence to Zhenhe Ma, E-mail: mazhenhe@163.com
Nonetheless, there is a lack of real-time continuous in vivo imaging techniques for detecting cerebral edema.

ICP monitoring is currently the clinical standard for the detection of such disease in vivo. However, ICP measurements exhibit a high degree of variability; ICP monitors are able to detect edema only if there is a sufficient increase in ICP. ${ }^{8}$ On the other hand, both brain water content (BWC) analysis and Evan's blue tracer can accurately reflect the degree of edema, but their disadvantage is that only in vitro measurements are allowed. ${ }^{9}$ Computed tomography is considered a common tool for clinical detection of cerebral edema, but it is not sensitive enough and consequently not suitable for detection at early stages. ${ }^{10,11}$ It is well documented that some pulse sequences of magnetic resonance imaging (MRI) can be applied to assess cerebral edema after ischemic stroke. ${ }^{12}$ Diffusion-weighted imaging (DWI) is capable of detecting changes in the apparent diffusion coefficient of water, to which cytotoxic edema is sensitive. ${ }^{13}$ The elevated values of $\mathrm{T} 2$-weighted resonance imaging (T2WI) reflect vasogenic edema. ${ }^{14}$ Although promising, DWI has the disadvantage of poor resolution; T2WI can detect cerebral edema only when ischemia has existed for a long time. $^{15}$

Optical coherence tomography (OCT) is a noninvasive method that allows volumetric imaging of biological tissues at high resolution and high speeds. ${ }^{16,17}$ Another OCT-based technique, calculating optical attenuation coefficient (OAC) from OCT signals, has been attracting the attention of scholars. ${ }^{18}$ Infrared light decays exponentially when penetrating tissues and its attenuation coefficient can be used to distinguish 
between different types of tissues, or different physiological states of the same tissue, and can be utilized to detect brain edema. ${ }^{19} \mathrm{~A}$ recent study showed that the OAC is affected by the water content of brain tissue. The transmittance of near-infrared light increases (i.e., OAC decreases) when edema occurs in brain tissue. Such change is due to the presence of acidic substances in edema cells, which increases the permeability of cell membranes. ${ }^{20}$ In another study, a "water poisoning model" was used, which was made by intraperitoneal injection of excess physiological saline into mice. Excessive water penetrates into the brain over time, and the water content of the brain cells gradually increases, resulting in cerebral edema. They used spectral-domain optical coherence tomography (SD-OCT) to detect OAC in different parts of the cerebral cortex and found that the average $\mathrm{OAC}$ of the cerebral cortex gradually decreased as cerebral edema progressed. ${ }^{21}$ The research team conducted another study that explored a focal model of edema in a mouse model of traumatic brain injury and observed changes in scatter correlated with edema formation around the impact site.

Edema caused by ischemic stroke can occur at specific locations or throughout either cerebral hemisphere. In order to better monitor the development of cerebral edema after stroke, it is necessary to provide sufficient imaging field-of-view (FoV) for the entire cortex. The swept-source optical coherence tomography (SS-OCT) developed in recent years has proven to have higher spectral resolution ${ }^{22}$ and greater light intensity ${ }^{23}$ than traditional SD-OCT. Therefore, SS-OCT has been widely used in clinical settings in areas, in areas such as ophthalmology ${ }^{24-26}$ and dermatology, ${ }^{27}$ and for imaging oral and nasal cavity tissues. ${ }^{28}$

In this paper, the cerebral edema status in middle cerebral artery occlusion (MCAO) rats over time was monitored for the first time using SS-OCT system. The imaging FoV was set large enough to cover the entire cortex. An optimized depth-resolved estimation (ODRE) method ${ }^{29}$ was applied to accurately calculate OAC of tissue at different depths. Cortical surface extraction and flattening were performed to facilitate the extraction of OAC signals at specific depths below the cortical surface. Then, an en face OAC maps were obtained by averaging the OACs in a specific depth range. The edema tissue was segmented from the OAC maps and the edema areas were quantified. After the collection of OCT data, both MRI and BWC measurements via the wet-dry methods were used to verify the presence of cerebral edema. This work extends the application of OCT and provides an option for cerebral edema in vivo detection after ischemic stroke.

\section{Method}

\subsection{Animal Model}

In this study, three-month-old Sprague-Dawley rats (males) were used, each weighing 250 to $280 \mathrm{~g}$. All procedures were performed in accordance with the Animal Ethics and Administrative Council of Northeastern University. All efforts were made to minimize animal suffering and to reduce the number of animals used. Surgical anesthesia was induced with sodium pentobarbital $(3 \%, 5 \mathrm{mg} / 100 \mathrm{~g}$, IP). During our experiment, the animal's body temperature was maintained at $36.8^{\circ} \mathrm{C}$ through a homoeothermic blanket system. The anesthetized rats were fixed on a stereotaxic apparatus (ST-5ND-C) with ear bars and a clamping device. The fur on the rats' heads was shaved, and the skin was cleaned with $0.9 \%$ sodium chloride physiological solution. The skin was cut along the midline of the skull, and the interparietal bone was exposed by pulling the skin to the sides. Next, subcutaneous tissue and the periosteum were cleaned. To obtain a clearer image, we ground the skull to the thickness of 0.1 to $0.2 \mathrm{~mm}$ using a $1.2-\mathrm{mm}$ diameter flat cranial drill. The grinding process needs to be performed carefully to avoid any damage, and the thickness should be uniform. The processed skull was almost transparent after adding saline. After the procedure, the rat was placed in the sample arm and prepared for data acquisition (baseline).

The model of cerebral ischemia used in our experiments was based on previously described Endothelin-1 (ET-1) induced MCAO model. ${ }^{30}$ The stereotaxic coordinates of the ET- 1 injection were $0.9 \mathrm{~mm}$ anterior, $5.2 \mathrm{~mm}$ lateral, and $8.7 \mathrm{~mm}$ ventral relative to bregma. A dose of $3 \mu 1 \mathrm{ET}-1$ (diluted to $60 \mathrm{pmol} / \mathrm{L}$ in $0.9 \%$ sodium chloride) was injected at $1 \mu \mathrm{l} / \mathrm{min}$. After injection, we waited for $3 \mathrm{~min}$ to ensure that the drug was fully absorbed. The needle was slowly removed and the rat was immediately placed under the sample arm to begin scanning. In the first $40 \mathrm{~min}$, we collected data every $10 \mathrm{~min}$. During 40 to $130 \mathrm{~min}$, the interval was $30 \mathrm{~min}$.

\subsection{System}

A wide FoV SS-OCT system, set up in our previous work, ${ }^{31}$ was used in this study, as shown in Fig. 1. The light source employed was an akinetic swept-wavelength laser source (MEMSVCSEL, Thorlabs Inc., Newton, New Jersey), which operates at $200-\mathrm{kHz}$ swept rate at a central wavelength of $1300 \mathrm{~nm}$ with a 100-nm bandwidth to provide an axial resolution of $\sim 7.5 \mu \mathrm{m}$ in air. The beam emitted by the SS was split into the sample arm and the reference arm by 90:10 ratio coupler (TW1300R2A2, Thorlabs Inc.). In the sample arm, an aiming beam was combined with another 99:1 coupler to guide the OCT imaging. A 50-mm focal length objective lens (AC254-050-C, Thorlabs Inc.) was used in the sample arm to achieve $\sim 16-\mu \mathrm{m}$ lateral resolution. A pair of XY galvanometric mirrors was used in the system to form 3-D scans. The fast scanner ( $x$ direction) was driven by a sawtooth waveform with a $200-\mathrm{Hz}$ frame rate. Each B-scan image contained $1000 \mathrm{~A}$-scans that occupied $80 \%$ duty

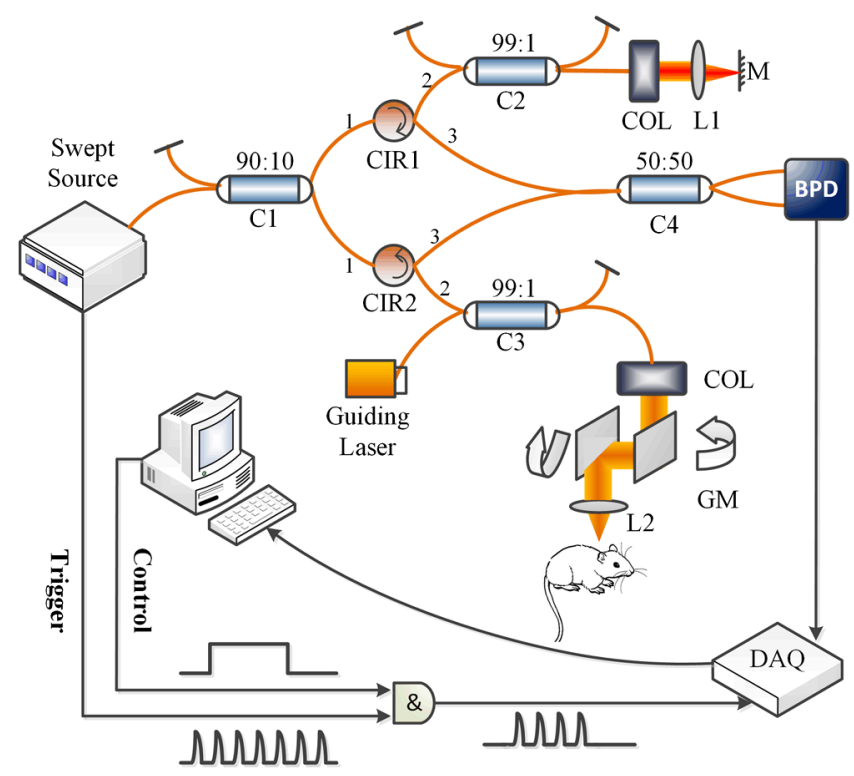

Fig. 1 Wide FoV SS OCT system. L1, L2: lens; C1, C2, C3, C4: fiber coupler; BPD: balanced photodetector; M: mirror; COL: collimator; CIR1, CIR2: circulator; GM: galvanometric mirror. 
cycle. The slow scanner ( $y$ direction) was driven by a step function waveform, which led to $800 \mathrm{~B}$-scan positions. The FoV is $12 \mathrm{~mm}(x$ direction $) \times 10 \mathrm{~mm}(y$ direction $)$, enough to cover the entire cerebral cortex of the rats. In order to control both the galvanometric mirror and the acquisition synchronization, we added an AND gate circuit to the system. An AND operation was performed to the triggering signal from the SS and the control signal (square wave), and the resultant signal was used to control data acquisition.

\subsection{OAC-Based Brain Edema Imaging}

\subsubsection{Elimination of confocal interference}

The intensity of OCT signals is affected by the distance between the detected position and the focal plane. The closer the distance, the stronger the signal intensity, on the contrary, the weaker. Such mechanism can destroy the exponential decay characteristics of infrared light and seriously affect the accurate calculation of OAC. Different positions on the rat skull have differing illumination rate due to physiological curvature. Therefore, the confocal interference must be eliminated before OAC calculation.

Recently, Faber et al. derived the general expression of the confocal axial point spread function (PSF), ${ }^{32,33}$ which is described as

$$
\begin{aligned}
& I(z) \propto h(z) \cdot e^{-2 \mu z}, \\
& h(z)=\left[\left(\frac{z-z_{c f}}{z_{R}}\right)^{2}+1\right]^{-1},
\end{aligned}
$$

where $\mu$ is the OAC, $z$ is the signal depth, the function $h(z)$ is the axial PSF, $z_{\mathrm{cf}}$ is the position of the confocal gate, and $z_{R}$ is the "apparent" Rayleigh length used to characterize the axial PSF.

$z_{R}=\alpha \pi n w_{0}^{2} / \lambda$

where $w_{0}$ is the minimum beam radius, $\lambda$ is the center wavelength of the light source, $n$ is the refractive index (we used $n=1.353), \alpha$ is used to distinguish specular reflection $(\alpha=1)$ from diffuse reflection $(\alpha=2)$. In this paper, $z_{R} \approx 417 \mu \mathrm{m}$. The influence of confocal characteristics could be removed by dividing the intensity of OCT signals by axial PSF $h(\mathrm{z}) .^{34}$

\subsubsection{Surface extraction and flattening}

In order to facilitate subsequent calculations, the cerebral cortex needs to be flattened to place the cortex surface of the brain at the same level. First, the skull surface of the sample was detected by using the moving summation window method proposed in the literature. ${ }^{35}$ Note that the cortex is $\sim 0.15 \mathrm{~mm}$ below the skull surface; the cortex surface was obtained by removing skull pixels. Then, a 3-D matrix was reconstructed from the cortex surface, extending a specific number of pixels vertically. In order to ensure a high signal-to-noise ratio, we selected a depth range of $3 \mathrm{~mm}$ for data analysis in phantom experiments and chose a range of $3.4 \mathrm{~mm}$ in the animal experiment. Noise floor exists in OCT signals. ${ }^{36}$ The presence of noise floor can cause OAC to be substantially underestimated, and the lower the signal-tonoise ratio, the greater the effect. Therefore, it should be eliminated in the manner of subtracting the noise floor from the original OCT data before OAC calculation.

\subsubsection{Calculation of optical attenuation coefficient}

The calculation of OAC from OCT signals is usually performed by fitting an exponential curve along OCT A-scans. However, this method requires a prelayered image and assumes that the medium in each layer is uniform. In practice, however, it is difficult to achieve precise stratification of different types of tissue. Recently, Vermeer et al. developed a depth-resolved estimation (DRE) method based on single scattering, ${ }^{37}$ which converts each pixel of an OCT signal into a corresponding OAC value. However, this method has a disadvantage. When there is a noninsignificant sum of signal that would have been reflected from past the full depth of the image, errors will appear and increase with depth.

In this study, we used an ODRE method proposed in the previous work. $^{29}$ This method can effectively solve the error problem caused by the DRE method and can accurately calculate OAC at any depth. Under the condition that the backscattered light reflected toward the photodetector of the OCT system is a fixed fraction of the attenuated light, each pixel in the OCT dataset is converted to a corresponding OAC value using the following relationship:

$\mu[z]=\frac{I[z]}{2 \Delta \sum_{i=z+1}^{N} I[i]+\frac{I[N]}{\mu[N]},}$

where $I[z]$ is the OCT signal of a pixel and $\mu[z]$ is its corresponding OAC, both at depth $z$ and $\Delta$ is the pixel size (usually related to the axial resolution of the OCT system). $N$ is the number of pixels within a limited depth. $I[N]$ is the OCT signal for the last point $N . \mu[N]$ in Eq. (4) is unknown. To determine it, a piece of data was taken from the end of the imaging depth and then fitted to an exponential curve. The fitting model is $y=a \cdot \exp (-2 \mu z)+b$. The fitting result $\mu$ is the average attenuation coefficient of this data and can be considered as the best approximation of $\mu[N] . b$ is the strength of the noise floor, which should be subtracted from the original OCT signal before the OAC calculation. OCT signals are generally highly noisy, so we averaged five adjacent A-scans before exponential fitting to increase the signal-to-noise ratio and then replace $I[N]$ with the fitted $y[N]$. After exponential fitting, we introduce the fitted $\mu$ into Eq. (4). When all the A-scan calculations are completed, a set of 3-D OAC matrices are obtained.

\subsection{Magnetic Resonance Image Acquisition}

A 3.0-T MRI system (Verio, Siemens Medical Solutions, Erlangen, Germany) was utilized with a special coil for animals. After anesthesia, a group of rats was placed in the prone position and the head of each was placed in the center of the coil for axial scanning. The MR parameters were as follows: axial T2WI turbo spin-echo (TR: $4000 \mathrm{~ms}$; TE: $111 \mathrm{~ms}$; flip angle: $150 \mathrm{deg}$; FoV: $70 \mathrm{~mm} \times 70 \mathrm{~mm}$; voxel size: $0.4 \mathrm{~mm} \times 0.3 \mathrm{~mm} \times 1.8 \mathrm{~mm}$; slice thickness: $1.8 \mathrm{~mm}$; number of slices: 10). Prototype software was used to carry out the imaging analysis on a workstation (Siemens Verio 3.0-T MR Leonardo 3682). The MRI acquisition time was set to $24 \mathrm{~h}$ after MCAO.

\subsection{Brain Water Content Analysis}

The presence of cerebral edema was verified by analyzing BWC via the wet-dry methods. After the collection of OCT data was completed, animals were killed and their brains were dissected 
out postmortem and cut into three pieces (right-left cerebral hemispheres and cerebellum). Brain samples were immediately weighed on an electronic analytical balance to obtain their wet weight. They were then dried in a gravity oven at $100^{\circ} \mathrm{C}$ for $24 \mathrm{~h}$ to obtain their corresponding dry weight. ${ }^{21}$ The percent of BWC was calculated with the following formula:

$\mathrm{BWC}=[($ wet weight - dry weight $) /$ wet weight $] \times 100 \%$.

\section{Result}

\subsection{Phantom Experiments}

To experimentally verify the accuracy of the ODRE method, a two-layered agar phantom with different concentrations of $\mathrm{TiO}_{2}$ particles was fabricated to mimic the gray and white matter of the rat cortex. The top and bottom layer contains $0.1 \mathrm{wt} \%$ and 0.05 wt $\%$ of $\mathrm{TiO}_{2}$, respectively. The thickness of each thin phantom was controlled to be around 1.2 to $1.3 \mathrm{~mm}$. The OCT intensity image and corresponding $\mathrm{OAC}$ image are shown in Figs. 2(a) and 2(b). The averaged A-scan profiles of both images are shown in Figs. 2(c) and 2(d).

We first used the exponential fitting method to calculate the OAC for each layer of the phantom. The fitting model is $\mathrm{y}=a \cdot e^{-2 \mu x}+b$, whose results are shown in Fig. 2(c). The fitted values for the top and bottom layers were 0.81 and $0.4 \mathrm{~mm}^{-1}$, and their average OACs obtained with the proposed method were 0.80 and 0.39 , respectively. The two types of results above turned out to match, which proves that the proposed method has high accuracy.

\subsection{In Vivo Rat Brain Imaging}

Figure 3(a) shows a typical OCT B-scan intensity image at baseline, and the corresponding OAC image was shown in Fig. 3(b). The B-scan intensity image and its corresponding OAC image at 130 min after MCAO are shown in (c) and (d), respectively.
Compared with the baseline, both the scattering and OAC decreased in deep layers at $130 \mathrm{~min}$. In order to better visualize cerebral edema, we need to identify the depth, where the difference between edema and normal tissue is maximum. The averaged OAC curves at both the baseline and $130 \mathrm{~min}$ from an area of $240 \times 240 \mu \mathrm{m}^{2}(20 \times 20$ pixels $)$ in the ischemia region are shown, respectively, in Fig. 2(d).

The solid blue and red lines represent the average OAC curve at the baseline and at $130 \mathrm{~min}$, respectively. The orange dotted curve represents the difference between two curves along the depth, which is denoted by the $y$ axis on the right side of the graph. In the range of 0.5 to $2.5 \mathrm{~mm}$, we can see that the OAC at $130 \mathrm{~min}$ is generally lower than that at baseline. Specifically, the difference between the two in the range of 0.7 to $1 \mathrm{~mm}$ is greatest. Such difference indicates an optimal contrast between edema and healthy tissue, so we reconstructed en face OAC images by averaging the OACs within this depth range. The reconstruction results are shown in Fig. 4.

From this figure, obviously, no abnormalities occurred at baseline. A shadow began to appear in the ischemic zone $20 \mathrm{~min}$ after the MCAO model was established, which was reflected by a decrease in OAC, indicating the presence of brain edema. With time, the shadow gradually deepened and the area gradually expanded, which indicates that the condition was deteriorating.

We segmented the shaded area from these en face OAC images manually. Take the $40 \mathrm{~min}$ OAC image as an example, the segmented area is denoted by the red dashed line in Fig. 5(a); the area of this shadow was calculated. The changes in brain edema area with time in three rats are shown in Fig. 5(b).

To further analyze the changes in OAC over time, we selected three $240 \times 240 \mu \mathrm{m}^{2}$ regions on the ipsilateral hemisphere and three on the contralateral hemisphere, as shown in red square 1, 2, 3 and blue square 1, 2, 3 in Fig. 5(a), respectively. The average OAC curves for each region are shown in Fig. 5(c). All values are reported as mean \pm standard error of the mean, $n=20 \times 20$. In contrast, we found that all OACs
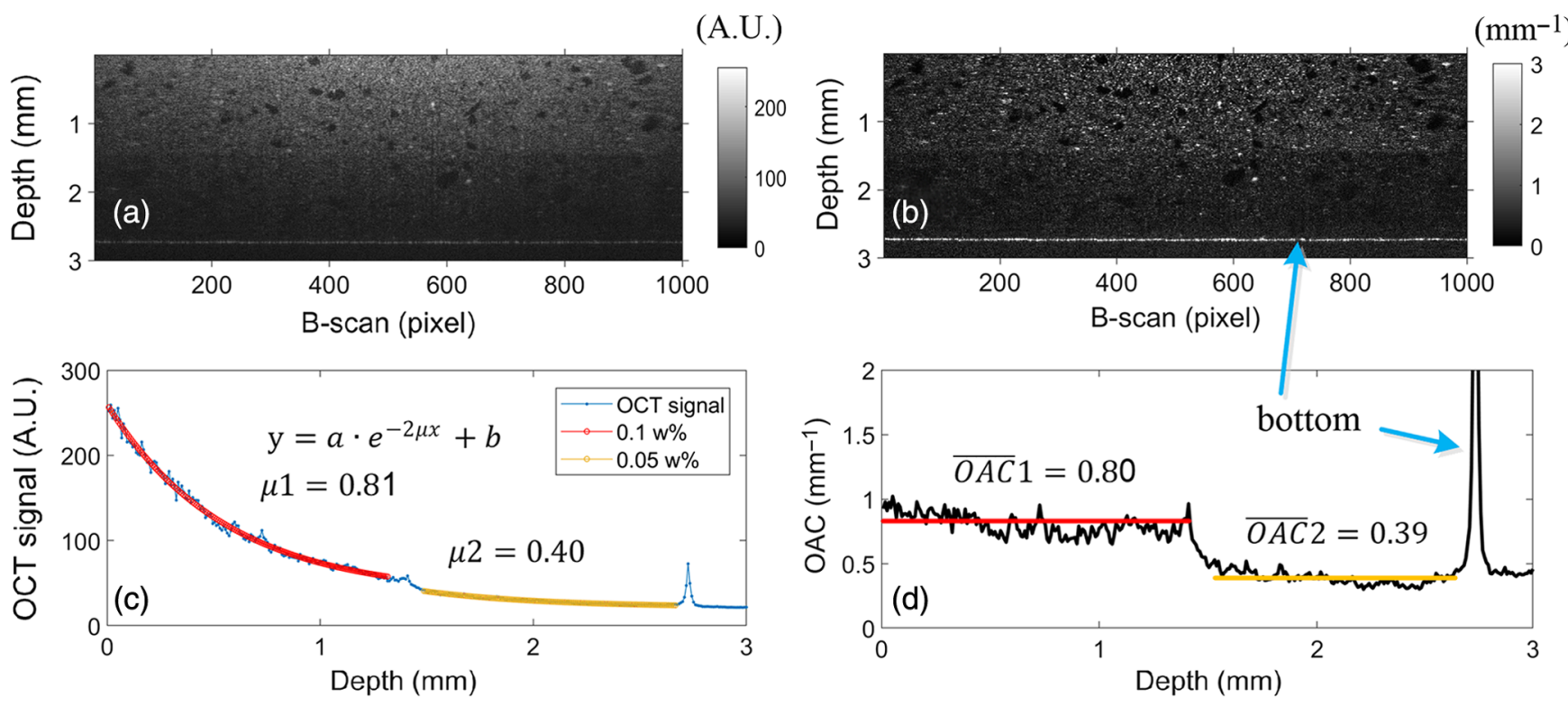

Fig. 2 (a) OCT intensity image and (b) the corresponding OAC image of a two-layered agar phantom. The top and bottom layer contains 0.1 and $0.05 \mathrm{wt} \%$ of $\mathrm{TiO}_{2}$, respectively. (c) The averaged A-scan profile of the intensity image with the result of exponential curve fitting. (d) The corresponding averaged OAC curve. The blue arrow indicates the bottom of the phantom. 

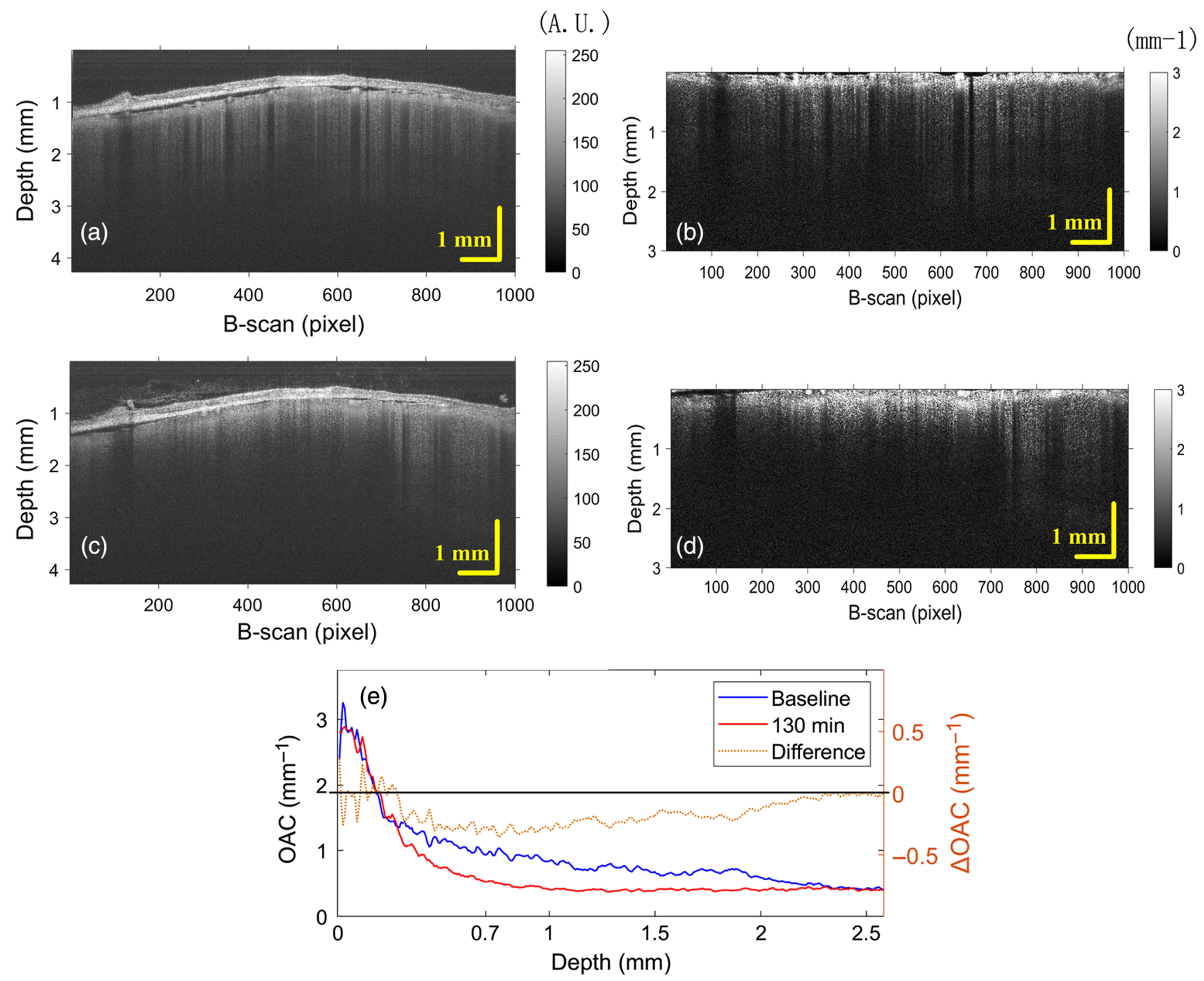

Fig. 3 (a), (c) Typical SS-OCT B-scan structural images at baseline and $130 \mathrm{~min}$ after MCAO; the corresponding OAC images are shown in (b) and (d). (e) The averaged A-scan OAC curves along the depth from an area of $240 \times 240 \mu \mathrm{m}^{2}$ in the ischemia region at the baseline and $130 \mathrm{~min}$, respectively, are shown. The orange dotted curve represents the difference between the two curves along the depth, which is denoted by the $y$ axis on the right of the graph.

of the ipsilateral hemisphere were significantly smaller than those of the contralateral hemisphere.

From Fig. 4, we also found that the OAC is smaller in the vessels. This is because the blood vessels are within about 300 to $500 \mu \mathrm{m}$ below the surface of the cortex, but the range we used for imaging was within 0.7 to $1 \mathrm{~mm}$. The flow of blood can greatly affect the scattering characteristics of the OCT signal on the back of the blood vessel. That is, the OAC value of the tissue on the back of the blood vessel is greatly reduced. During the ischemic process, we found that the positions of the blood vessels changed over time. We believe this is an occupancy effect caused by cerebral edema (Fig. 6).

Figure 7 shows a representative MRI coronal position T2WI image at $24 \mathrm{~h}$ after MCAO. The area with high signal intensity (white) in the ischemic hemisphere indicates cerebral edema, which is consistent with the SS-OCT result.

We performed a BWC test immediately after the MRI scan. The results show that the water content of the ipsilateral hemisphere was higher than that of the contralateral hemisphere
(79.5\% versus $77.7 \%)$, and there was a significant difference between the two $(* *$ represents $p<0.01, n=3$ ). Relevant BWC measurements confirmed the presence of cerebral edema (Fig. 8).

\section{Discussion}

Cerebral edema is one of the major complications of ICVD and the main cause of death within a week after its onset. ${ }^{9}$ Studies have shown that when brain edema is formed, swollen brain tissue squeezes peripheral blood vessels, causing peripheral brain tissue ischemia. There can be a vicious circle of "cerebral edema-cerebral ischemia-re-edema-reischemia," which will eventually trigger life-threatening cerebral herniation. ${ }^{38} \mathrm{~A}$ real-time, high-resolution imaging technology for in vivo detection of brain edema may play an active role in the research of treatment programs for this disease.

This study attempted to monitor the cerebral edema status in the whole cortex of MCAO rats over time by using SS-OCT 


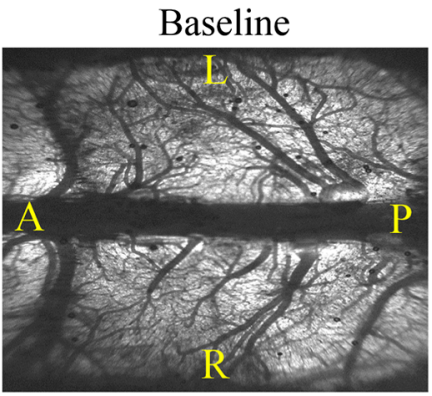

$70 \mathrm{~min}$

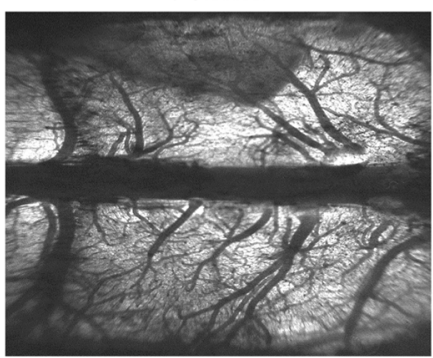

$20 \mathrm{~min}$

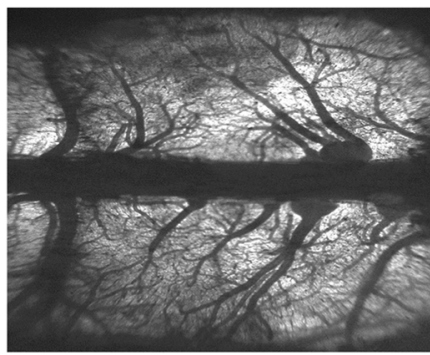

$100 \mathrm{~min}$

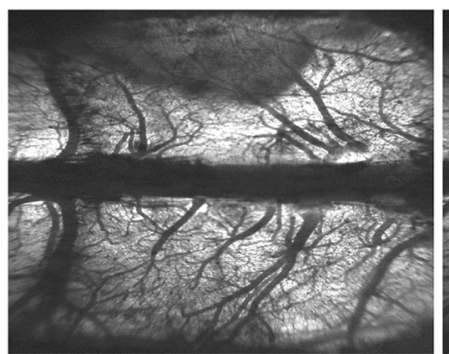

$40 \min$

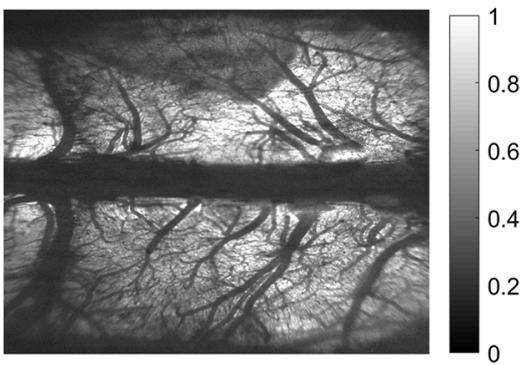

$130 \mathrm{~min}$

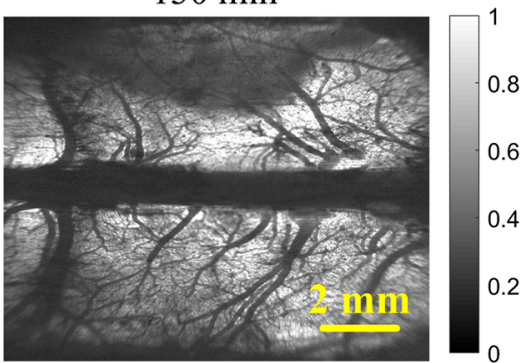

Fig. 4 En face OAC images at different times, the shaded area indicates the formation of cerebral edema.
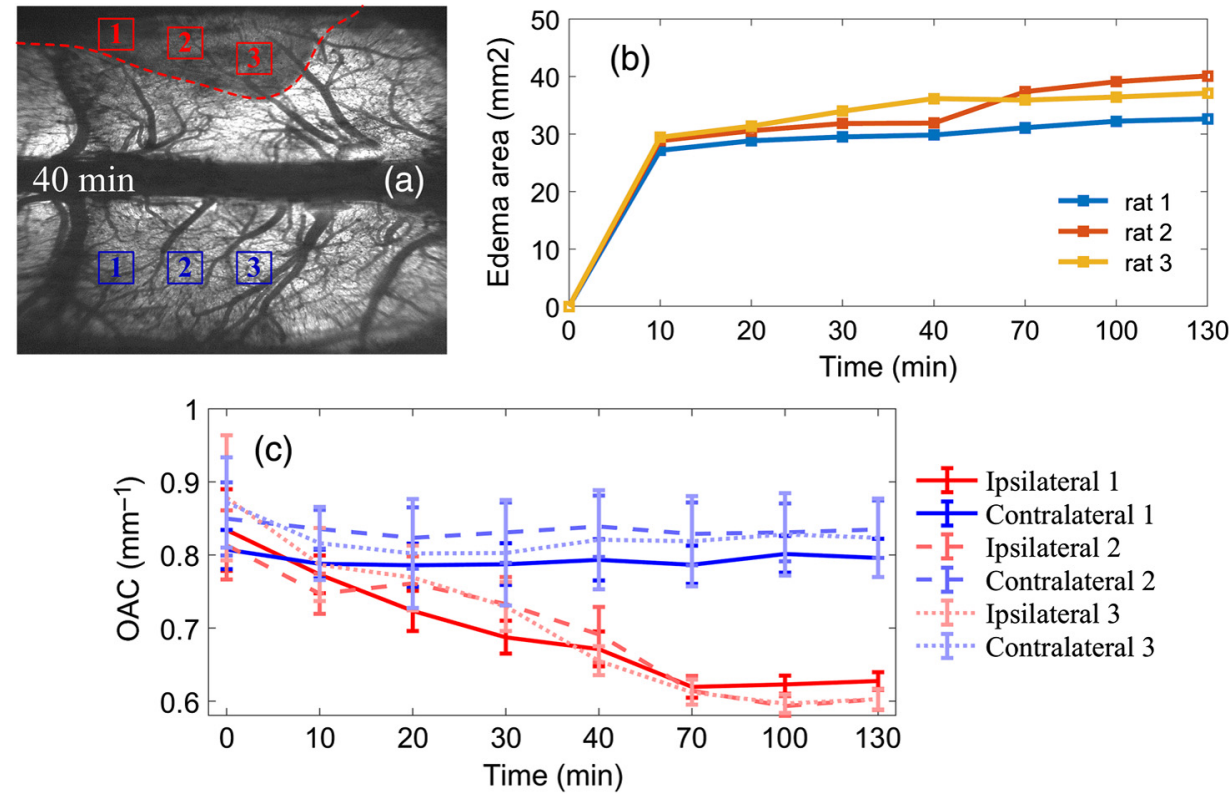

Fig. 5 (a) En face OAC image at $40 \mathrm{~min}$, the red dotted line marks the area where the OAC dropped significantly. Three $240 \times 240 \mu \mathrm{m}^{2}$ regions on the ipsilateral hemisphere and three on the contralateral hemisphere were selected, as shown by the red and blue boxes, respectively. (b) The changes in brain edema area with time in three rats. (c) The OAC of each box changed over time. All values are reported as the mean \pm standard error of the mean $(n=3)$.

system, and some preliminary results are reported in this paper. The rodent brain has a natural curvature, with a significant depth difference $(>5 \mathrm{~mm})$ between the anterior cerebral artery side and the MCA side. SS-OCT has a larger penetration depth and a smaller sensitivity fall-off along the depth. These advantages enable SS-OCT to better cope with rugged samples in wide FoV imaging.

The MCAO model used in this study was achieved by injecting a fixed dose of ET-1 at a specific location. The model simulated progressive vascular occlusion and reperfusion, which was very similar to real stroke in the human..$^{39}$ In addition, the model had the advantages of simple operation, small trauma, and high success rate. When cerebral edema occurred, the acidic substances produced in the cells increased the permeability of the cell membrane. The transmittance of infrared light increased with the increase of water content of brain tissue while the OAC decreases accordingly. Therefore, edema tissue and normal tissue can be distinguished with OAC. 


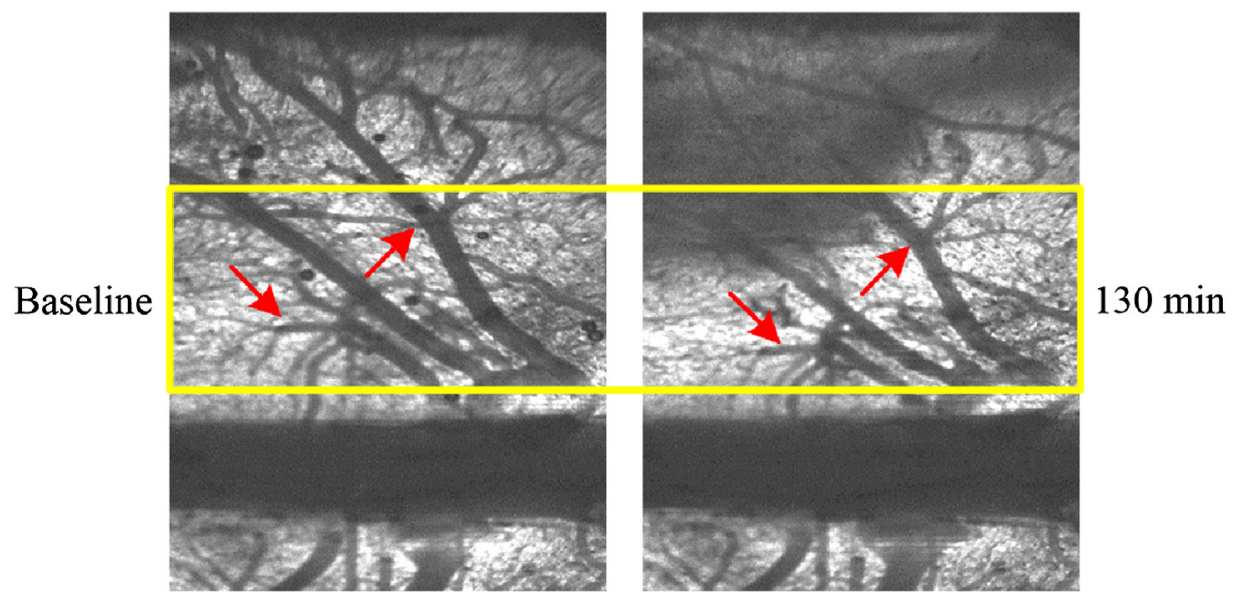

Fig. 6 Schematic diagram of vascular displacement. The yellow rectangle represents a region where vascular displacement is observable, while the red arrows indicate the displaced blood vessel.
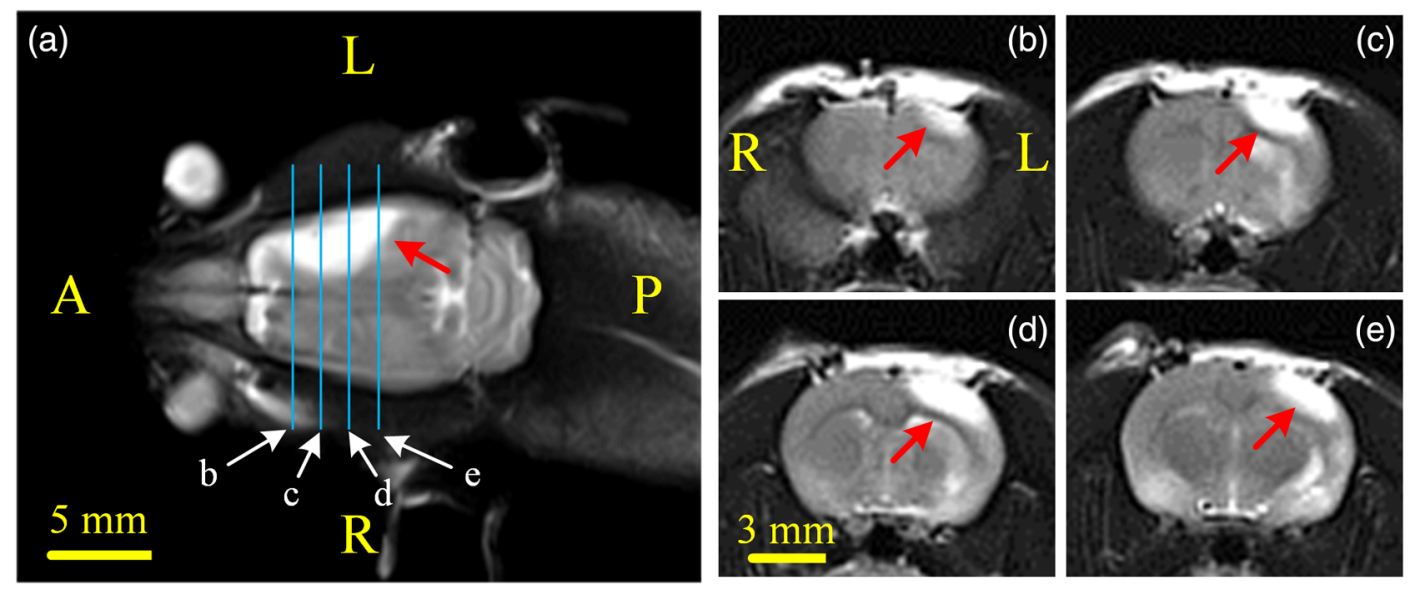

Fig. $7 \mathrm{MRI}$ T2WI image at $24 \mathrm{~h}$ after MCAO. (a) Transverse image; red arrow refers to the edema area. (b)-(e) Coronal image of the position of each solid blue line in (a).

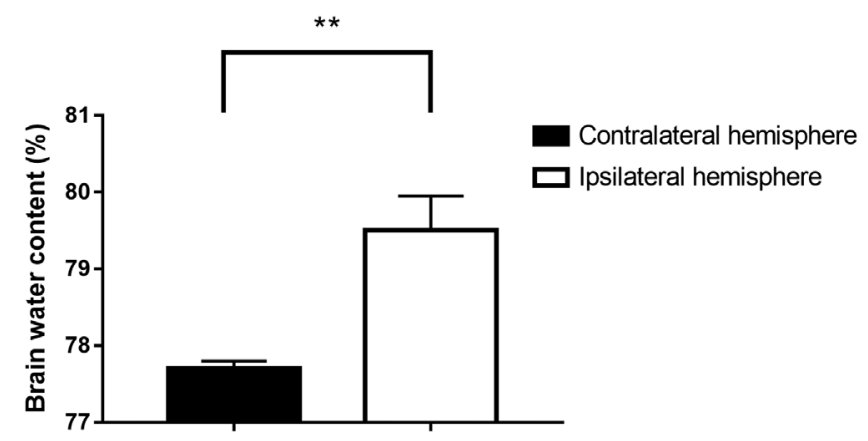

Fig. 8 BWC measurements. Error bars represent standard error ( ${ }^{* \star}$ represents $p<0.01, n=3$ ).

Before OAC estimation, we performed data processing on OCT signals, during which the influence of the confocal function was taken into account. Based on an article by Faber et al., we estimated the confocal axial PSF, in which $Z_{\mathrm{R}}$ is the only parameter. If $Z_{\mathrm{R}}$ is overestimated, the OAC value will be greater than the true one; otherwise, it will be less than it. The thickness of the rat's cerebral cortex is about $1.5 \mathrm{~mm},{ }^{40}$ and we selected data within the depth range of $3.4 \mathrm{~mm}$ below the cortical surface for data analysis, which covers all of gray matter. The DRE method proposed by Vermeer et al. will produce error that increases with depth. The edema area is located deep in the cortex, so the error produced by the DRE algorithm can have a huge impact on the OAC. The ODRE method can cope with this error problem properly and ensures accurate detection of image information on edema.

There are many blood vessels in the superficial layer of the cerebral cortex; the blood has stronger infrared light scattering, so the shallow OAC value is larger. In depths of the cortex, the blood vessels are sparse and the OAC value is small. In addition, the blood flow can greatly affect the scattering characteristics of 
the OCT signal on the back of the blood vessel, causing a "tail artifact" (Ref. 41). Therefore, the tissue on the back of blood vessels has a lower OAC than avascular regions. The curve in Fig. 3 was obtained by averaging the OACs of a specific area $(240 \times 240 \mu \mathrm{m})$; it is very difficult to completely avoid blood vessels, so a similar attenuation result occurs. This result is consistent with those of Ref. 21.

Compared with other studies, the OAC obtained by our system is small, which may be due to the sensitivity of the system. Some studies have shown that SD-OCT is prone to insufficient spectral resolution, and its sensitivity decreases rapidly with imaging depth. Compared with SD-OCT, SS-OCT has higher spectral resolution, and its sensitivity fall-off is almost negligible. $^{23}$ Therefore, SS-OCT signal intensity changes less with depth, i.e., the OAC is smaller.

Srinivasan et al. monitored brain injury progression based on intrinsic optical scattering properties of cortical tissue. ${ }^{42}$ They found that the penetration depth of OCT signals was reduced after reperfusion in the region destined for infarction. The OCT receives backscattered light from the sample, and the reduction in the imaging depth of OCT is associated with a combination of factors. One is the increased scattering of shallow tissue, which is consistent with our previous study. ${ }^{29}$ Another factor is the reduction in scattering of deep tissues. Infrared light scatters and is absorbed as it passes through biological tissue. Some studies show that the largest part of an OAC value might be contributed by scattering. ${ }^{43,44}$ Thus, the reduction in scattering could indicate an increase in permeability. Phantom experiments have shown that OCT can produce images at deeper locations if there is a strong reflective medium at the bottom of the sample. This shows that the decrease in OCT signal intensity sometimes does not mean that the permeability of the light is weakened but rather represents an enhancement of light permeability.

In our previous study, ${ }^{22}$ we found that blood flow immediately decreased after ET-1 injection. But, at this time, OAC did not change significantly. After a period of ischemia, the OAC in the ischemic area began to decrease significantly, and a low signal area appeared. As the low signal area gradually enlarged, the surrounding blood vessels were squeezed and displaced. Therefore, we believe that the "shadow" in Fig. 4 was caused by edema, not by a decrease in blood flow. In addition, during the ischemic process, we found that the positions of the blood vessels changed over time. We believe this is an occupancy effect caused by cerebral edema. The edema area detected by SS-OCT had a high similarity in position and shape to that obtained by MRI. BWC measurements also provided evidence that there is indeed edema in the rat brain.

There are still deficiencies in this study. One limitation is that physiological parameters were not monitored during experiments. Neglect of physiological parameters may affect the repeatability of the experiment. Another limitation is that MRI and BWC measurements were performed significantly later than OCT imaging due to certain constraints. This may result in changes in the physiological states of animals during the delay, which can weaken the verification of OCT results. The focus of this paper, however, is to propose a method for detecting brain edema in small animals as an option for in vivo brain edema research.

\section{Conclusion}

Cerebral ischemia can induce severe brain edema, resulting in changes in the optical properties of swollen brain cells, which can be detected by the OCT. Compared with other methods for brain edema detection, SS-OCT enables real-time high-resolution imaging in vivo, which is desirable for the basic research of ischemic cerebral edema; therefore, it is much likely to play an important role in the development of related drugs or in treatment options.

\section{Disclosures}

The authors declare that there are no conflicts of interest related to this article.

\section{Acknowledgments}

This work was supported in part by the National Natural Science Foundation of China (Grant Nos. 61771119 and 61901100), Hebei Provincial Natural Science Foundation of China (Grant Nos. H2018501087 and H2019501010). Fundamental Research Funds for the Central Universities (Grant Nos. N182304008, N172304034, and N172304029).

\section{References}

1. M. Faul et al., Traumatic Brain Injury in the United States: Emergency Department Visits, Hospitalizations, and Deaths 2002-2006, Centers for Disease Control and Prevention, National Center for Injury Prevention and Control, 2010, http://www.cdc. gov/traumaticbraininjury/tbi_ed .html.

2. C. Ayata and A. H. Ropper, "Ischaemic brain oedema," J. Clin. Neurosci. 9(2), 113-124 (2002).

3. M. Walberer et al., "Aggravation of infarct formation by brain swelling in a large territorial stroke: a target for neuroprotection?" J. Neurosurg. 109, 287-293 (2008).

4. D. Z. Wang, D. S. Nair, and A. V. Talkad, "Acute decompressive hemicraniectomy to control high intracranial pressure in patients with malignant MCA ischemic strokes," Curr. Treat. Opt. Cardiovasc. Med. 13(3), 225-232 (2011).

5. K. Vahedi et al., "Early decompressive surgery in malignant infarction of the middle cerebral artery: a pooled analysis of three randomised controlled trials," Lancet Neurol. 6(3), 215-222 (2007).

6. O. M. Arnaout et al., "Decompressive hemicraniectomy after malignant middle cerebral artery infarction: rationale and controversies," Neurosurg. Focus 30(6), E18 (2011).

7. E. M. Manno et al., "The effects of mannitol on cerebral edema after large hemispheric cerebral infarct," Neurology 52(3), 583-583 (1999).

8. A. Marmarou, "Pathophysiology of traumatic brain edema: current concepts," Acta Neurochir. Suppl. 86, 7, Springer, Vienna (2003).

9. A. Slivka, E. Murphy, and L. Horrocks, "Cerebral edema after temporary and permanent middle cerebral artery occlusion in the rat," Stroke 26(6), 1061-1066 (1995).

10. A. J. Yoo et al., "Validating imaging biomarkers of cerebral edema in patients with severe ischemic stroke," J. Stroke Cerebrovasc. Dis. 22(6), 742-749 (2013).

11. C. Cristia et al., "The association between a quantitative computed tomography (CT) measurement of cerebral edema and outcomes in post-cardiac arrest-a validation study," Resuscitation 85(10), 13481353 (2014).

12. K. S. Hong et al., "Diffusion changes suggesting predominant vasogenic oedema during partial status epilepticus," Seizure 13(5), 317-321 (2004).

13. M. D. Phillips and R. A. Zimmerman, "Diffusion imaging in pediatric hypoxic ischemia injury," Neuroimaging Clin. North Am. 9(1), 41-52 (1999).

14. J. Badaut et al., "Temporal and regional evolution of aquaporin-4 expression and magnetic resonance imaging in a rat pup model of neonatal stroke," Pediatr. Res. 62(3), 248-254 (2007).

15. A. Obenaus and S. Ashwal, "Magnetic resonance imaging in cerebral ischemia: focus on neonates," Neuropharmacology 55(3), 271-280 (2008). 
16. R. K. Wang, "Optical microangiography: a label-free 3-D imaging technology to visualize and quantify blood circulations within tissue beds in vivo," IEEE J. Sel. Top. Quantum Electron. 16(3), 545-554 (2010).

17. Z. Ma et al., "Quantification of cerebral vascular perfusion density via optical coherence tomography based on locally adaptive regional growth," Appl. Opt. 57(35), 10117-10124 (2018).

18. D. J. Faber et al., "Quantitative measurement of attenuation coefficients of weakly scattering media using optical coherence tomography," Opt. Express 12(19), 4353-4365 (2004).

19. D. Liang et al., "Cytotoxic edema: mechanisms of pathological cell swelling," Neurosurg. Focus 22, E2 (2007).

20. J. R. Thiagarajah, M. C. Papadopoulos, and A. S. Verkman, "Noninvasive early detection of brain edema in mice by near-infrared light scattering," J. Neurosci. Res. 80(2), 293-299 (2005).

21. C. L. R. Rodriguez et al., "Decreased light attenuation in cerebral cortex during cerebral edema detected using optical coherence tomography," Neurophotonics 1(2), 025004 (2014).

22. J. Liu et al., "Whole-brain microcirculation detection after ischemic stroke based on swept-source optical coherence tomography," $J$. Biophotonics 12, e201900122 (2019).

23. J. Xu et al., "Wide field and highly sensitive angiography based on optical coherence tomography with akinetic swept source," Biomed. Opt. Express 8(1), 420-435 (2017).

24. I. Grulkowski et al., "Retinal, anterior segment and full eye imaging using ultrahigh speed swept source OCT with vertical-cavity surface emitting lasers," Biomed. Opt. Express 3(11), 2733-2751 (2012).

25. I. Grulkowski et al., "High-precision, high-accuracy ultralong-range swept-source optical coherence tomography using vertical cavity surface emitting laser light source," Opt. Lett. 38(5), 673-675 (2013).

26. A. H. Dhalla et al., "Simultaneous swept source optical coherence tomography of the anterior segment and retina using coherence revival," Opt. Lett. 37(11), 1883-1885 (2012).

27. W. J. Choi and R. K. Wang, "Volumetric cutaneous microangiography of human skin in vivo by VCSEL swept-source optical coherence tomography," Quantum Electron. 44(8), 740-745 (2014).

28. W. J. Choi and R. K. Wang, "In vivo imaging of functional microvasculature within tissue beds of oral and nasal cavities by swept-source optical coherence tomography with a forward/side-viewing probe," Biomed. Opt. Express 5(8), 2620-2634 (2014).

29. J. Liu et al., "Optimized depth-resolved estimation to measure optical attenuation coefficients from optical coherence tomography and its application in cerebral damage determination," J. Biomed. Opt. 24(3), 035002 (2019).

30. J. Liu et al., "Hemodynamic changes in a rat parietal cortex after endothelin-1-induced middle cerebral artery occlusion monitored by optical coherence tomography," J. Biomed. Opt. 21(7), 075014 (2016).

31. J. Liu et al., "Whole-brain microcirculation detection after ischemic stroke based on swept-source optical coherence tomography," $J$. Biophotonics 12(10) (2019).

32. T. G. Van Leeuwen, D. J. Faber, and M. C. Aalders, "Measurement of the axial point spread function in scattering media using single-mode fiber-based optical coherence tomography," IEEE J. Sel. Top. Quantum Electron. 9(2), 227-233 (2003).

33. F. J. van der Meer et al., "Quantitative optical coherence tomography of arterial wall components," Laser Med. Sci. 20(1), 45-51 (2005).
34. G. T. Smith et al., "Automated, depth-resolved estimation of the attenuation coefficient from optical coherence tomography data," IEEE Trans. Med. Imaging 34(12), 2592-2602 (2015).

35. U. Baran, Y. Li, and R. K. Wang, "In vivo tissue injury mapping using optical coherence tomography based methods," Appl. Opt. 54(21), 6448-6453 (2015).

36. G. Liu et al., "A comparison of Doppler optical coherence tomography methods," Biomed. Opt. Express 3(10), 2669-2680 (2012).

37. K. A. Vermeer et al., "Depth-resolved model-based reconstruction of attenuation coefficients in optical coherence tomography," Biomed. Opt. Express 5(1), 322-337 (2013).

38. P. K. Manwaring et al., "Intracranial electrical impedance tomography: a method of continuous monitoring in an animal model of head trauma," Anesth. Analg. 117, 866-875 (2013).

39. C. F. Chang et al., "Hyperbaric oxygen therapy for treatment of postischemic stroke in adult rats," Exp. Neurol. 166(2), 298-306 (2001).

40. A. Belin-Rauscent et al., "From impulses to maladaptive actions: the insula is a neurobiological gate for the development of compulsive behavior," Mol. Psychiatr. 21(4), 491-499 (2016).

41. U. Baran et al., "Tail artifact removal in OCT angiography images of rodent cortex," J. Biophotonics 10(11), 1421-1429 (2017).

42. V. J. Srinivasan et al., "Multiparametric, longitudinal optical coherence tomography imaging reveals acute injury and chronic recovery in experimental ischemic stroke," PLoS One 8(8), e71478 (2013).

43. S. J. Matcher, M. Cope, and D. T. Delpy, "In vivo measurements of the wavelength dependence of tissue-scattering coefficients between 760 and $900 \mathrm{~nm}$ measured with time-resolved spectroscopy," Appl. Opt. 36(1), 386-396 (1997).

44. M. Hohmann et al., "Extension of depth-resolved reconstruction of attenuation coefficients in optical coherence tomography for slim samples," Proc. SPIE 9792, 97920P (2015).

Jian Liu, is a lecturer at the School of Control Engineering, Northeastern University at Qinhuangdao, China. His main research directions are optical coherence tomography, digital image processing, and ischemic cerebrovascular disease. He has published more than 10 academic papers and also taken out 3 patents.

Yao $\mathrm{Yu}$ is a lecturer at the School of Control Engineering, Northeastern University at Qinhuangdao, China. She was a postdoctoral associate in the Thrombosis and Haemostasis Lab group at Imperial College, London (2013-2014), and received her doctorate degree in clinical medicine research (investigative science) in 2013 from Imperial College, London. Her main research interests are thrombosis, vascular diseases, and optical coherence tomography.

Zhenhe $\mathrm{Ma}$ is an associate professor and mentor of graduate students at the School of Control Engineering, Northeastern University at Qinhuangdao, China. He was a postdoctoral fellow at Oregon Health \& Science University (2009-2010). His main research directions are optical coherence tomography, infrared spectroscopy, and Raman spectroscopy. He has published more than 70 academic papers, including over $40 \mathrm{SCl}$ searches and $20 \mathrm{El}$ searches, and he has also taken out 8 patents.

Biographies for the other authors are not available. 